1 Long-term outcomes with balloon-expandable and self-expandable prostheses

2 in patients undergoing transfemoral transcatheter aortic valve implantation for

3

4

5

6

$7 \quad{ }^{1}$ Equally contributing

8

9

\section{severe aortic stenosis} Switzerland

\footnotetext{
*Corresponding author:
}

N/A.

René Vollenbroich ${ }^{\mathrm{a}, *, 1}$, PeterWenaweser ${ }^{\mathrm{a}, \mathrm{d}, 1}$, Annina Macht ${ }^{\mathrm{a}}$, Stefan Stortecky ${ }^{\mathrm{a}}$, Fabien Praz ${ }^{\mathrm{a}}$, Martina Rothenbühler ${ }^{b}$, Eva Roost ${ }^{c}$, Lukas Hunziker ${ }^{a}$, Lorenz Räber ${ }^{a}$, Stephan Windecker ${ }^{\mathrm{a}}$, Thomas Pilgrim ${ }^{\mathrm{a}}$

${ }^{a}$ Department of Cardiology, Swiss Cardiovascular Center Bern, University Hospital, Bern, Switzerland

${ }^{\mathrm{b}}$ Clinical Trials Unit, Department of Clinical Research, and Institute of Social and Preventive Medicine (ISPM), University of Bern, Switzerland

${ }^{c}$ Department of Cardiovascular Surgery, Swiss Cardiovascular Center, University Hospital, Bern,

${ }^{\mathrm{d}}$ Heart Clinic Hirslanden Zurich, Switzerland

All authors take responsibility for all aspects of the reliability and freedom from bias of the data presented and their discussed interpretation.

Department of Cardiology, Bern University Hospital, University of Bern, Freiburgstrasse 8, CH-3010 Bern, Switzerland. E-mail address: rene.vollenbroich@insel.ch (R. Vollenbroich).

\title{
Acknowledgement of grant support
}


Potential conflicts of interest

Peter Wenaweser received lecture and proctoring fees from Medtronic, Edwards Lifesciences, and Boston Scientific. Fabien Praz received proctoring fees of Edwards Lifesciences. Stephan Windecker received research grants to the institution from Abbott, Amgen, Biotronik, Boston Scientific, St Jude Medical, Terumo and Bayer. Thomas Pilgrim received research grants to the institution from Symetis/Boston Scientific, Edwards Lifesciences, and Biotronik and received speaker fees from Boston Scientific and Biotronik. All other authors report no potential conflict of interests.

\section{Keywords}

Transcatheter aortic valve implantation (TAVI), Valvular heart disease, Outcome, Structural valve deterioration, Self-expanding prosthesis, Balloon-expandable prosthesis

\section{Tables and 2 Figures}

Table 1: Baseline clinical characteristics.

Table 2: Echocardiographic imaging characteristics.

Figure 1: (A) Cumulative incidence including landmark analysis of all-cause mortality according to transcatheter aortic valve type up to 5 years of follow-up. (B) Cumulative incidence including landmark analysis of cardiac mortality according to transcatheter aortic valve type up to 5 years of follow-up. (C) Cumulative incidence including landmark analysis of major stroke according to transcatheter aortic valve type up to 5 years of follow-up.

Figure 2: Cumulative incidence of structural valve deterioration up to 5 years of follow-up. 
$47 \quad$ Background

Data on long-term outcomes in patients undergoing transcatheter aortic valve implantation (TAVI) is

49

50

51

52 scarce.

$\underline{\text { Methods }}$

We investigated long term outcomes of consecutive patients undergoing TAVI with balloon- and selfexpandable bioprostheses (Edwards SAPIEN (ESV), Edwards Lifesciences Inc., Irvine, CA, USA; Medtronic Corevalve system (MCS), Medtronic Inc., Minneapolis, MN, USA).

\section{$\underline{\text { Results }}$}

Among 628 patients (mean age $82.4 \pm 5.8$ years, 55\% female), 489 (77.8\%) underwent transfemoral TAVI. 309 (63.2\%) patients received a MCS prosthesis, whereas 180 (36.8\%) patients were treated with an ESV prosthesis. The median duration of follow-up amounted to 5.2 years (range 3.4-8.3 years). All-cause mortality did not differ between the two groups (MCS 46.9\%, ESV 53.4\%, Cl 95\%: RR $1.21[0.93-1.57], P=0.15)$, whereas cardiac mortality was higher in the ESV cohort after 5 years of follow-up (MCS 35.1\%, ESV 45.4\%, CI 95\%: RR 1.37 [1.01-1.86], $P=0.04$ ). Structural valve deterioration, which was on average diagnosed 41.9 months (range 18-60 months) after TAVI, occurred in 8 cases $(1.6 \%)$, resulting in one repeat intervention.

\section{Conclusions}

While half of all patients died within 5 years after TAVI with no significant differences in all-cause mortality, structural valve deterioration was documented in b2\% of cases. 


\section{INTRODUCTION}

Transcatheter aortic valve implantations (TAVI) are rapidly expanding towards the low risk spectrum of patients with severe aortic stenosis. Randomized controlled trials showed comparable safety and efficacy of both, self- and balloon-expandable prostheses, as compared to surgical aortic valve replacement [1-3]. Regarding the use of TAVI in younger patients, the question of long-term outcomes and in particular of valve durability becomes of major importance. However, there is a significant lack of data regarding these factors, which can also be seen as directories regarding the decision making in favor of TAVI or surgical aortic valve replacement (SAVR) in patients with a lower operative risk profile. The aim of the present analysis was to evaluate the long-term outcomes regarding the performance of the two most widely used TAVR systems: the balloon-expandable Edwards SAPIEN valve (ESV) (Edwards Lifescience Inc., Irvine, CA, USA) and the self-expandable Medtronic Corevalve system (MCS) (Medtronic Inc., Minneapolis, MN, USA) in patients undergoing TAVI for severe symptomatic aortic valve stenosis.

\section{METHODS}

\section{Study population}

Between July 2007 and January 2013, all patients undergoing TAVI at the Swiss Cardiovascular Center of Bern University Hospital in Switzerland were consecutively recorded in a prospective registry held at the Clinical Trials Unit of the University of Bern in Switzerland. Inclusion criteria consisted of a) symptomatic, severe aortic stenosis (AS) with an echocardiographic mean gradient $>40 \mathrm{~mm} \mathrm{Hg}$ or a calculated aortic valve area $<1 \mathrm{~cm}^{2}$ and b) age $\geq 80$ years with a high operative risk score (logistic European System for Cardiac Operative Risk Evaluation (EuroSCORE) >15\%). Patients $<80$ years of age were eligible if at least one of the following comorbid conditions were present: previous cardiac surgery, liver cirrhosis, chronic pulmonary disease (forced expiratory volume $<1 \mathrm{l} / \mathrm{s}$ ), severe pulmonary hypertension, porcelain aorta, history of mediastinal radiotherapy, severe connective tissue disease with contraindication for surgery, or frailty. Additionally, anatomical 
prerequisites consisted of an aortic annulus diameter in the range of 18 to $27 \mathrm{~mm}$ and a vascular access site suitable for transfemoral TAVI. Exclusion criteria included degenerated aortic valve prostheses and severe aortic regurgitation in the absence of AS. An interdisciplinary team of cardiac surgeons and interventional cardiologists reviewed all cases and formed a consensus on treatment allocation (TAVI or SAVR). The registry as well as the study have been approved by the local cantonal ethics committee and comply with the Declaration of Helsinki. All patients enrolled in the database provided written informed consent.

\section{Definitions and procedures}

Patients undergoing TAVI underwent comprehensive multimodal assessment using transthoracic and transesophageal echocardiography, right and left heart catheterization, and contrast computed tomography. TAVI was performed according to standard protocols via transfemoral approach using both balloon-expandable ESV (Sapien THV and XT) and self-expandable MCS. Device selection was based on anatomical and technical characteristics as described previously [4]. Pre- and postdilatation were performed according to the operators' discretion. Postinterventional antithrombotic and antiplatelet treatment was prescribed according to the discretion of the operator. For definitions of outcome variables see Supplemental File 1. Procedural success was defined as device success in the absence of major adverse cardiovascular and cerebral events during the first $48 \mathrm{~h}$ after device implantation. Device success was defined according to VARC-2 criteria. Bioprosthetic valve dysfunction, including valve deterioration, thrombosis, and endocarditis, was defined according to the consensus statement from the European Association of Percutaneous Cardiovascular Interventions (EAPCI).

\section{Data collection}

Demographic characteristics, imaging parameters, hemodynamic measurements, and procedural variables were prospectively recorded in a web-based database. All patients underwent sweep 
follow-up between April and November 2017 which was performed by means of standardized telephone interviews. In addition, medical records, discharge summaries, and documentation of hospitalization were systematically collected from general practitioners, referring cardiologists as well as referring hospitals for verification of clinical endpoints. For a validated calcification score analysis [5], measurements were done at theHU-850 threshold in Contrast CT images. All endpoints were defined according to the updated version of the Valve Academic Research Consortium (VARC2) definitions [6], and adjudicated by a clinical event committee, which consists of interventional cardiologists and cardiac surgeons from different institutions.

\section{Statistical analysis}

Continuous data are reported as mean \pm standard deviation (SD) if their distribution is approximately normal and as median/range otherwise. The means were compared using analysis of variance and differences in medians were analysed with Mann-Whitney test. Categorical variables are expressed as number of patients (\% of patients). Survival was estimated using the Kaplan-Meier method and differences in estimates were compared by means of the log-rank test. The at-risk time span was derived from the date of intervention and the last available data of the patient, determined either by the last follow-up, the time of death, or information coming from referring hospitals and/or practitioners. Survival estimates were calculated using univariate and multivariate Cox proportional hazard models including landmark analyses. Reported are crude hazard ratios (HR; with 95\% confidence intervals) with $\mathrm{p}$-values from Wald chi-square tests, or continuity correct risk ratios with p-values from Fisher's exact tests. P-values $<0.05$ were considered statistically significant. For adjusted analyses, baseline and pre-TAVI characteristics were included that showed a difference between the two groups with a p-value b 0.1 (TAVI device, sex, body mass index, previous CABG, previous stroke or TIA, prior permanent pacemaker, EuroScore, aortic valve area, LV ejection fraction, and calcification score). All analyses were performed with Stata version 14 (StataCorp, College Station, TX, USA). 
No extramural funding was used to support this work.

RESULTS

Among 628 patients (mean age $82.4 \pm 5.8$ years, $54.6 \%$ female), 489 patients $(77.8 \%)$ underwent

transfemoral TAVI for native aortic valve stenosis. Patients undergoing transapical ( $N=124,19.7 \%)$

or trans-subclavian ( $\mathrm{N}=9,1.4 \%) \mathrm{TAVI}$, as well as patients with a transcatheter-valve-in-surgical-valve procedure $(N=6,1 \%)$ were excluded from the present analysis. $309(63.2 \%)$ patients were treated with a MCS whereas 180 (36.8\%) patients received an ESV (ESV THV in 27 (5.5\%) cases, ESV XT in 153 (31.3\%) cases). Baseline clinical characteristics at the time of intervention are summarized in Table 1. Both, patients in the MCS and the ESV cohort, were comparable with respect to cardiovascular risk factors, clinical features, symptom status, and preinterventional antithrombotic therapy. While more female patients underwent implantation of a MCS (MCS $46.3 \%$ vs. ESV $35 \%, P=0.0148$ ), patients in the ESV cohort had more frequently experienced a previous stroke or transient ischemic attack (TIA) as compared with MCS patients (ESV $10.8 \%$ vs. MCS 4.8\%, P = 0.0136). Echocardiographic imaging characteristics are outlined in Table 2. No significant preinterventional differences between the two treatment arms could be noted except a higher left ventricular ejection fraction (LVEF) within the ESV group (ESV $56.6 \%$ vs. MCS $51.8 \%$ vs., $P=0.0004$ ). Furthermore, measurements from left/right heart catherization were comparable between the two groups (Supplemental Table 1).

\section{Procedural outcomes}

Procedural data are depicted in Supplemental Table 2. Procedure time did not significantly differ between MCS and ESV and took on average $67.5 \mathrm{~min}(\mathrm{P}=0.52)$. Implantation of a MCS valve required more contrast dye (MCS $266.7 \pm 102.7 \mathrm{ml}$, ESV $225.7 \pm 96 \mathrm{ml}, \mathrm{P} \leq 0.001$ ) and was less frequently performed with a balloon predilatation as compared with the implantation of an ESV 
mean aortic valve gradient (MCS 7.2 $\pm 3.7 \mathrm{~mm} \mathrm{Hg}$, ESV $8.5 \pm 4.0 \mathrm{~mm} \mathrm{Hg}, \mathrm{P}=0.0003$ ) whereas the need for permanent pacemaker implantation was higher in the MCS cohort (MCS $29.8 \%$ vs. ESV $14.4 \%, P=0.0001)$. Postprocedural moderate to severe aortic regurgitation occurred more frequently among patients treated with a MCS valve (MCS 19.1\% vs. ESV $4.5 \%, \mathrm{P} \leq 0.0001$ ). The mean hospital duration was $9.1(7.2 \pm 12.3)$ days with a longer duration for patients treated with a MCS prosthesis (MCS $9.1(8.4 \pm 12.4)$ days, ESV $8.3(7.3 \pm 11.1)$ days, $P=0.02)$. In 11 cases, all of which occurred within the MCS cohort $(3.6 \%, \mathrm{P}=0.01)$, the implantation of more than one valve in series was required.

\section{Clinical outcomes}

Comparisons of clinical outcomes are descriptive. The median duration of follow-up amounted to 5.2 years (range 3.4-8.3 years). None of the patients was lost to follow-up. Event rates with crude hazard ratios for all major clinical endpoints according to VARC through 30 days, 3 years, and 5 years are provided in Supplemental Table 3. All-cause mortality throughout 5 years of follow-up did not differ between the two groups (MCS 46.9\%, ESV 53.4\%, RR 1.21 [0.93-1.57], $P=0.15$ ) whereas cardiac mortality was higher in the ESV cohort, taking effect after 3 years (30 days: MCS 3.9\%, ESV 5.6\%, RR 1.59 [0.67-3.75], $P=0.29 ; 3$ years: MCS 21.6\%, ESV 24.5\%, RR $1.18[0.8-1.75], P=0.4 ; 5$ years: MCS 35.1\%, ESV 45.4\%, RR 1.37 [1.01-1.86], P = 0.04). Fig. 1 shows cumulative event rates for all-cause mortality, cardiac mortality, and major stroke throughout 5 years stratified for MCS and ESV and including landmark analyses ( 0 to 30 days, 31 days to 5 years) with the aforementioned described significant difference in long-term cardiac mortality $(P=0.04)$ between the two groups. The landmark analyses as such did not show any further significant differences between the two valve types (Supplemental Table 4). Adjusted univariable analyses showed an association between all-cause mortality and female gender ( $\mathrm{HR} 0.73,95 \% \mathrm{Cl} 0.57-0.95 ; \mathrm{P}=0.0183)$, previous CABG (HR 1.60, 95\% Cl 1.03-2.49; $\mathrm{P}=0.0355)$, logistic EuroScore (HR 1.02, 95\% Cl 1.01-1.03; P b 0.001), and LVEF (HR 0.99, 95\% Cl 0.98-1.00; $\mathrm{P}=0.0052$ ), between cardiac mortality and the implantation of an 
ESV (HR 1.37, 95\% Cl 1.01-1.86; P=0.0409), logistic EuroScore (HR 1.03, 95\% Cl 1.02-1.04; P <0.001), and LVEF (HR 0.98, 95\% Cl 0.97-0.99; $\mathrm{P}=0.0006)$, whereas major stroke was associated with previously occurred strokes or transitoric ischemic attacks (HR 3.27, 95\% $\mathrm{Cl} 1.23-8.72 ; \mathrm{P}=0.0177$ ). Univariable and multivariable adjusted analyses are illustrated in Supplemental Tables 5 and 6.

\section{Echocardiographic follow-up and time-related valve safety}

Post-procedural echocardiographic data relate to the last available transthoracic echocardiographic follow-up performed at the university center or at an outpatient cardiology center. After three years, echocardiographic data amounted to $72 \%$ of cases; after five years echocardiographic follow-up data was available in $65 \%$ of cases. While mean and peak aortic valve (AV) gradients as well as LVEF were higher in the ESV cohort (mean AV gradient: MCS $8.85 \pm 4.75 \mathrm{~mm} \mathrm{Hg}$; ESV $10.25 \pm 4.52 \mathrm{~mm} \mathrm{Hg}, \mathrm{P}=$ 0.0033; peak AV gradient: MCS $16.23 \pm 9.4 \mathrm{~mm} \mathrm{Hg}$; ESV $18.59 \pm 8.69 \mathrm{~mm} \mathrm{Hg}, \mathrm{P}=0.0277$; LVEF: MCS $55.55 \pm 12.43 \mathrm{~mm} \mathrm{Hg}$; ESV $57.83 \pm 10.94 \mathrm{~mm} \mathrm{Hg}, \mathrm{P}=0.0543$ ), severe pulmonary hypertension (MCS 40.4\% vs. ESV 26.9\%, $\mathrm{P}=0.0335$ ) and moderate or severe aortic regurgitation (MCS $19 \%$ vs. ESV 9\%, $\mathrm{P}$ $=0.0055)$ were more frequently observed in patients treated with a MCS valve. Regarding relevant aortic regurgitation, no significant change could be seen over time after TAVI in both, MCS and ESV treated patients ( $\mathrm{MCS}: \mathrm{P}=0.1384, \mathrm{ESV}: \mathrm{P}=0.0621$ ). The degree and changes in aortic regurgitation before and after treatment have been depicted in Supplemental Figs. 1 and 2 . In total, 8 cases (1.6\%) of structural valve deterioration (SVD) (3 MCS (1\%), 5 ESV (2.8\%)) occurred during the follow-up time. On average, prosthetic SVD was diagnosed 41.9 months (range 18-60 months) after TAVI. Moderate SVD occurred in 7 cases (ESV: 4 (2.2\%), MCS: $3(1 \%))$, whereas severe SVD was only found in one patient (ESV, 0.6\%). Details are shown in Supplemental Tables 7 and 8 as well as in Fig. 2. A repeat procedure due to SVD was performed in only one case 4.6 years after implantation of an ESV XT $26 \mathrm{~mm}$ (mean AV gradient $64 \mathrm{~mm} \mathrm{Hg}$, aortic valve area (AVA) $0.6 \mathrm{~cm}^{2}$ ) with a successful valve-invalve procedure using a MCS valve. All other cases of SVD were treated conservatively. In addition to the SVD case, valve-related repeat interventions were performed in another four patients $(0.1 \%)$. In 
two patients, who were primarily treated with a MCS-valve, a balloon dilatation of the transcatheter valve was performed due to relevant paravalvular regurgitation 13 days and 14 days after the index procedure. One patient with a MCS valve developed severe paravalvular aortic regurgitation after 1.3 years and was treated with another MCS prosthesis. Another patient was diagnosed with an aorto-right ventricular fistula 1.3 months after implantation of an ESV prosthesis resulting in a fistula occlusion with a coil [7]. In total, two cases of prosthetic valve endocarditis were diagnosed. One with an ESV XT $26 \mathrm{~mm}$ valve 2.6 years after implantation and the other one with an ESV XT $23 \mathrm{~mm}$ 4.8 years after implantation. No case of manifest prosthetic valve thrombosis occurred during the follow-up.

\section{DISCUSSION}

We present long-term clinical outcomes of patients with a symptomatic severe AS treated with transfemoral TAVI using either a balloon-expandable (ESV) or a self-expandable (MCS) prosthesis. The key findings can be summarized as follows: (1) $>50 \%$ of patients died within 5 years after TAVI; there were no differences in all-cause mortality and major stroke between patients treated with either a balloon-expandable ESV or a self-expandable MCS prosthesis; (2) Structural valve deterioration occurred in $<2 \%$ of survivors and was diagnosed on average 3.5 years after the indexprocedure; (3) Repeat interventions for prosthetic heart valve related problems were rare.

Our results of a high efficacy of both, the balloon-expandable ESV and the self-expandable MCS valve, can be confirmed through various studies [8-10]. However, valve-specific drawbacks have been previously described as well. In patients treated with a MCS prosthesis, we observed a higher need for permanent pacemaker implantation ( $29.8 \%$ vs. $14.4 \%$ at 30 days, $P=0.0001)$, which was consistent with previous reports [11-14]. This fact is most likely due to the deeper extension of the valve into the left ventricular outflow tract in addition to the self-expanding nature of its frame applying constant pressure on the atrioventricular conductance system. Regarding rates of atrioventricular conduction disturbance and potential impact on long-term mortality, conflicting 
evidence exists. While data from our cohort suggested that preprocedural pacemaker implantation does not adversely affect clinical outcomes, data of the PARTNER study showed that the presence of a pacemaker (pre- or periprocedural) was independently associated with increased 1-yearmortality $[15,16]$. However, further technical developments, such as adjustments of the valve frame and additional modifications of the catheter, which allows a more accurate positioning of the valve, may further reduce the likelihood of a pacemaker dependency $[17,18]$. In addition, patients treated with MCS more commonly had paravalvular regurgitation as compared to patients treated with ESV (19.1\% vs. 4.5\%, P $\leq 0.001)$, which has previously been associated with worse long-term clinical outcomes [19]. Our results are in line with reported rates of relevant AR after TAVI with early generation devices ranging from $15 \%$ to $20 \%$ [20-24]. Most of the cases of no/mild aortic regurgitation at baseline that worsened were worsening from mild to moderate aortic regurgitation. Improved valve positioning and stabilisation resulting in predictable implantation depth in combination with refinements of the prosthesis with skirts, cuffs, and seals, have significantly reduced the rate of paravalvular regurgitation $[25,26]$. Despite the higher rates of moderate to severe paravalvular regurgitation, valve in series procedures, and permanent pacemaker implantation in the MCS group, there was no excess mortality in this cohort, even though all of these complications have been associated with worse outcomes as described above. This paradoxon may be partially explained by the moderate sample size of this study as well as by "background" events of death occurring in octogenerians as already hypothesized by the one year results of the CHOICE trial [27]. well as the cardiac mortality rate of $21.6 \%$ for the MCS and $24.5 \%$ for the ESV cohort at 3 -year follow-up is within the range of previous reports, albeit at the lower end [28-31]. Outcome data beyond 3 years in terms of comparison of the two most widely used TAVI systems is scarce. Bouleti et al. showed a 5 -year event-free survival rate of $28 \% \pm 4 \%$, however, the study cohort was small ( $\mathrm{N}$ $=123$ ) and in $>90 \%$ of patients, an ESV prosthesis was used [32]. In the study of Tarantini and 
colleagues, 171 patients were treated (MCS: $N=87, E S V: N=84$ ) with an overall survival rate of 44.9\% at 5 years without a difference between valve types [33]. Data of the UK TAVI Registry with an almost balanced implantation rate between MSC and ESV prostheses, presented a 5 year all-cause mortality rate of 53.1\% being in line with our findings (MCS 46.9\%, ESV 53.4\%). Valve type differences at 5 years as well as data on cardiac mortality were not presented. Our results showed a statistically higher cardiac mortality in the ESV group (MCS 35.1\% vs. ESV 45\%, $\mathrm{P}=0.04$ ) taking effect after 3 years. Crude cardiac mortality rates of patients treated with an ESV prosthesis were lower as compared with the 5-year results from the PARTNER trial (45.4\% vs. $57.5 \%$ ) [30]. Of note, no relevant difference in calcification volume could be found within the two cohorts. Due to the observational nature of this single center study these results have to be interpreted with caution. Notwithstanding, and with the knowledge that a lot of morbidities unrelated to cardiovascular disease heavily contribute to death in the long-term, this effect requires further scrutiny and needs to be considered for further analyses comparing the two valve systems. The incidence of adverse events including stroke at 3 and at 5 years were comparable to other reports and showed no differences between the valve types.

The low incidence of time-related valve safety events according to VARC is reassuring and comparable to other long-term TAVI studies [29,34,35]. Structural valve deterioration occurred in 8 patients (1.6\%) in both, patients treated with an ESV or MCS-valve. Referring to the consensus statement from the European Association of Percutaneous Cardiovascular Interventions [36], moderate SVD occurred more frequently as compared with severe SVD, underlined by data from the NOTION trial [37]. While reported rates of structural valve deterioration in surgically implanted aortic valve prostheses requiring reoperation range from $6-47 \%$ by 12 to 29 years after implantation, reports of transcatheter valve durability are needed to safely expand TAVR to the low risk spectrum of younger patients [38-42]. The observation of subclinical leaflet thrombosis (SLT) has recently raised concerns and may affect long-term clinical outcomes, in particular rates of 
cerebrovascular events [41,42]. Further research is crucial in order to evaluate if actual rates of bioprosthetic valve dysfunction also relate to newer generation valves.

The present analysis has to be interpreted against the background of several limitations. First, the number of patients included into the analysis was modest. Conversely, no patient was lost to clinical follow-up and reports on long-term outcome of patients undergoing TAVI are scarce. Second, data was acquired at a single center, thus not being generalizable to institutions with different referral patterns. Third, allocation to treatment with MCV and ESV was non-randomized; differences in clinical outcomes are therefore open to bias. Fourth, current data on long-term follow-up includes treatment with older generation valves resulting in a possible impact on generalizability.

Furthermore, the assessment of long-term structural valve deterioration might be limited in high-risk populations with rather high mortality rates in the early TAVI era. Additionally, the lack of uniformity of echocardiography and the low follow-up data of echocardiography over time might have introduced a bias in addition to a possible bias of underestimation of valve thrombosis in the absence of routine multisliced computed tomography in SVD patients. However, the analyses represent treatment decisions and outcomes of consecutive patients as encountered in routine clinical practice.

\section{CONCLUSION}

More than $50 \%$ of patients undergoing TAVI died within 5 years of the procedure with no significant differences in all-cause mortality between MCS and ESV. Structural valve deterioration was documented in $<2 \%$ of patients. 


\section{REFERENCES}

1. M.J. Reardon, N.M. Van Mieghem, J.J. Popma, et al., Surgical or transcatheter aortic-valve replacement in intermediate-risk patients, N. Engl. J. Med. 376 (2017) 1321-1331.

2. M.B. Leon, C.R. Smith, M.J. Mack, et al., Transcatheter or surgical aortic-valve replacement in intermediate-risk patients, N. Engl. J. Med. 374 (2016) 1609-1620.

3. V.H. Thourani, S. Kodali, R.R. Makkar, et al., Transcatheter aortic valve replacement versus surgical valve replacement in intermediate-risk patients: a propensity score analysis, Lancet 387 (2016) 2218-2225.

4. P. Wenaweser, T. Pilgrim, N. Roth, et al., Clinical outcome and predictors for adverse events after transcatheter aortic valve implantation with the use of different devices and access routes, Am. Heart J. 161 (2011) 1114-1124.

5. H. Jilaihawi, R.R. Makkar, M. Kashif, et al., A revised methodology for aortic-valvar complex calcium quantification for transcatheter aortic valve implantation, Eur. Heart J. Cardiovasc. Imaging 15 (2014) 1324-1332.

6. A.P. Kappetein, S.J. Head, P. Genereux, et al., Updated standardized endpoint definitions for transcatheter aortic valve implantation: the Valve Academic Research Consortium-2 consensus document, J. Am. Coll. Cardiol. 60 (2012) 1438-1454.

7. T. Pilgrim, B. Meier, P. Wenaweser, Aorto-right ventricular fistula after transfemoral aortic valve implantation, J. Invasive Cardiol. 22 (2010) E30-E31.

8. M.B. Leon, C.R. Smith, M. Mack, et al., Transcatheter aortic-valve implantation for aortic stenosis in patients who cannot undergo surgery, N. Engl. J. Med. 363 (2010) 1597-1607.

9. R.R. Makkar, G.P. Fontana, H. Jilaihawi, et al., Transcatheter aortic-valve replacement for inoperable severe aortic stenosis, N. Engl. J. Med. 366 (2012) 1696-1704.

10. S. Beurtheret, N. Karam, N. Resseguier, et al., Outcomes after transthoracic, peripheral vascular and transfemoral transcatheter aortic valve implantation: a propensity analysis from France TAVI Registry, J. Am. Coll. Cardiol. 71 (2018) A990. 
11. A. Chieffo, G.L. Buchanan, N.M. Van Mieghem, et al., Transcatheter aortic valve implantation with the Edwards SAPIEN versus the Medtronic CoreValve Revalving system devices: a multicenter collaborative study: the PRAGMATIC Plus Initiative (Pooled-RotterdAm-MilanoToulouse In Collaboration), J. Am. Coll. Cardiol. 61(2013) 830-836.

12. H. Jilaihawi, D. Chin, M. Vasa-Nicotera, et al., Predictors for permanent pacemaker requirement after transcatheter aortic valve implantation with the CoreValve bioprosthesis, Am. Heart J. 157 (2009) 860-866.

13. J. Baan Jr., Z.Y. Yong, K.T. Koch, et al., Factors associated with cardiac conduction disorders the CoreValve prosthesis, Am. Heart J. 159 (2010) 497-503.

14. H. Eltchaninoff, A. Prat, M. Gilard, et al., Transcatheter aortic valve implantation: early results of the FRANCE (FRench Aortic National CoreValve and Edwards) registry, Eur. Heart J. 32 (2011) 191-197.

15. L. Buellesfeld, S. Stortecky, D. Heg, et al., Impact of permanent pacemaker implantation on clinical outcome among patients undergoing transcatheter aortic valve implantation, J. Am. Coll. Cardiol. 60 (2012) 493-501.

16. J.M. Dizon, T.M. Nazif, P.L. Hess, et al., Chronic pacing and adverse outcomes after transcatheter aortic valve implantation, Heart 101 (2015) 1665-1671.

17. D. Tchetche, T. Modine, B. Farah, et al., Update on the need for a permanent pacemaker after transcatheter aortic valve implantation using the CoreValve (R) Accutrak system, Eurolntervention 8 (2012) 556-562.

18. A.S. Petronio, J.-M. Sinning, N. Van Mieghem, et al., Optimal implantation depth and adherence to guidelines on permanent pacing to improve the results of transcatheter aortic valve replacement with the Medtronic CoreValve system: the CoreValve prospective, international, post-market ADVANCE-II study, J. Am. Coll. Cardiol. Intv. 8 (2015) 837-846. 
19. M. Abdel-Wahab, R. Zahn, M. Horack, et al., Aortic regurgitation after transcatheter aortic valve implantation: incidence and early outcome. Results from the German transcatheter aortic valve interventions registry, Heart 97 (11) (2011) 899-906 (hrt. 2010.217158).

20. C.R. Smith, M.B. Leon, M.J.Mack, et al., Transcatheter versus surgical aortic-valve replacement in high-risk patients, N. Engl. J. Med. 364 (2011) 2187-2198.

21. C. Tamburino, D. Capodanno, A. Ramondo, et al., Incidence and predictors of early and late mortality after transcatheter aortic valve implantation in 663 patients with severe aortic stenosis clinical perspective, Circulation 123 (2011) 299-308.

22. K. Takagi, A. Latib, R. Al-Lamee, et al., Predictors of moderate-to-severe paravalvular aortic regurgitation immediately after corevalve implantation and the impact of postdilatation, Catheter. Cardiovasc. Interv. 78 (2011) 432-443.

23. D. Détaint, L. Lepage, D. Himbert, et al., Determinants of significant paravalvular regurgitation after transcatheter aortic valve implantation: impact of device and annulus discongruence, J. Am. Coll. Cardiol. Intv. 2 (2009) 821-827.

24. M.-A. Clavel, J.G. Webb, P. Pibarot, et al., Comparison of the hemodynamic performance of percutaneous and surgical bioprostheses for the treatment of severe aortic stenosis, J. Am. Coll. Cardiol. 53 (2009) 1883-1891.

25. R.K. Binder, S. Stortecky, D. Heg, et al., Procedural results and clinical outcomes of transcatheter aortic valve implantation in Switzerland: an observational cohort study of Sapien 3 versus Sapien XT transcatheter heart valves, Circ. Cardiovasc. Interv. 8 (2015), e002653.

26. S.L. Noble, S. Stortecky, D. Heg, et al., Comparison of procedural and clinical outcomes with Evolut R versus Medtronic CoreValve: a Swiss TAVI registry analysis, Eurolntervention 12 (2017) e2170-e2176. 
27. M. Abdel-Wahab, F.-J. Neumann, J. Mehilli, et al., 1-Year outcomes after transcatheter aortic valve replacement with balloon-expandable versus self-expandable valves: results from the CHOICE randomized clinical trial, J. Am. Coll. Cardiol. 66(2015) 791-800.

28. G.M. Deeb, M.J. Reardon, S. Chetcuti, et al., 3-Year outcomes in high-risk patients who underwent surgical or transcatheter aortic valve replacement, J. Am. Coll. Cardiol. 67 (2016) $2565-2574$.

29. M. Barbanti, A.S. Petronio, F. Ettori, et al., 5-Year outcomes after transcatheter aortic valve implantation with CoreValve prosthesis, JACC Cardiovasc. Interv. 8 (2015)1084-1091.

30. M.J.Mack, M.B. Leon, C.R. Smith, et al., 5-Year outcomes of transcatheter aortic valve replacement or surgical aortic valve replacement for high surgical risk patients with aortic stenosis (PARTNER 1): a randomised controlled trial, Lancet 385 (2015) 2477-2484.

31. M. Gilard, H. Eltchaninoff, P. Donzeau-Gouge, et al., Late outcomes of transcatheter aortic valve replacement in high-risk patients: the FRANCE-2 registry, J. Am. Coll. Cardiol. 68 (2016) $1637-1647$.

32. C. Bouleti, D. Himbert, B. lung, et al., Long-term outcome after transcatheter aortic valve implantation, Heart 101 (2015) 936-942.

33. G. Tarantini, P.A.M. Purita, A. D'Onofrio, et al., Long-term outcomes and prosthesis performance after transcatheter aortic valve replacement: results of self-expandable and balloon-expandable transcatheter heart valves, Ann. Cardiothorac. Surg. 6 (2017) 473-483.

34. S.R. Kapadia, M.B. Leon, R.R. Makkar, et al., 5-Year outcomes of transcatheter aortic valve replacement compared with standard treatment for patients with inoperable aortic stenosis (PARTNER 1): a randomised controlled trial, Lancet. 385 (2015) 2485-2491.

35. J. Rodés-Cabau, J.G.Webb, A. Cheung, et al., Long-term outcomes after transcatheter aortic valve implantation: insights on prognostic factors and valve durability from the Canadian multicenter experience, J. Am. Coll. Cardiol. 60 (2012) 1864-1875. 
36. D. Capodanno, A.S. Petronio, B. Prendergast, et al., Standardized definitions of structural deterioration and valve failure in assessing long-term durability of transcatheter and surgical aortic bioprosthetic valves: a consensus statement from the European Association of Percutaneous Cardiovascular Interventions (EAPCI) endorsed by the European Society of Cardiology (ESC) and the European Association for Cardio-Thoracic Surgery (EACTS), Eur. J. Cardiothorac. Surg. 52 (2017) 408-417.

37. L. Sondergaard, Data Presented at: Euro PCR 2018 Congress; Paris, France, 2018.

38. P.H. Neville, M.R. Aupart, F.F. Diemont, A.L. Sirinelli, E.M. Lemoine, M.A. Marchand, Carpentier-Edwards pericardial bioprosthesis in aortic or mitral position: a 12-year experience, Ann. Thorac. Surg. 66 (1998) S143-S147.

39. P.S.U. Mykén, O. Bech-Hansen, A 20-year experience of 1712 patients with the Biocor porcine bioprosthesis, J. Thorac. Cardiovasc. Surg. 137 (2009) 76-81.

40. P. Biglioli, N. Spampinato, A. Cannata, et al., Long-term outcomes of the Carpentier-Edwards pericardial valve prosthesis in the aortic position: effect of patient age, J.Heart Valve Dis. 13 (Suppl. 1) (2004) S49-S51.

41. R.R. Makkar, G. Fontana, H. Jilaihawi, et al., Possible subclinical leaflet thrombosis in bioprosthetic aortic valves, N. Engl. J. Med. 373 (2015) 2015-2024.

42. T. Chakravarty, L. Søndergaard, J. Friedman, et al., Subclinical leaflet thrombosis in surgical and transcatheter bioprosthetic aortic valves: an observational study, Lancet 389 (2017) $2383-2392$. 
442 Table 1: Baseline clinical characteristics.

443 Depicted are means \pm SD with $p$-values from t-tests or counts (\%) with p-values from Fisher's tests

444 (two categories) or chi-square tests (more than two categories). BMI = Body mass index; CABG=

445 Coronary artery bypass grafting; GFR = Glomerular Filtration Rate; MI=Myocardial infarction;

446 IQR=Interquartile range; NYHA=New York Heart Association; $\mathrm{PCl}=$ Percutaneous coronary

447 intervention; TIA = Transient ischemic attack; STS = Society of Thoracic Surgeons.

\begin{tabular}{|c|c|c|c|c|}
\hline & \multirow{2}{*}{$\begin{array}{l}\text { Overall } \\
N=489\end{array}$} & \multirow{2}{*}{$\begin{array}{l}\text { Medtronic } \\
\text { CoreValve }\end{array}$} & \multirow{2}{*}{$\begin{array}{l}\text { Edwards } \\
\text { Sapien } \\
N=180\end{array}$} & \multirow[t]{2}{*}{$\begin{array}{l}P \\
\text { value }\end{array}$} \\
\hline & & & & \\
\hline \multicolumn{5}{|l|}{ Demographics } \\
\hline Age, years & $82.9 \pm 5.2$ & $82.8 \pm 5.1$ & $83.0 \pm 5.6$ & 0.7534 \\
\hline Female gender, n (\%) & $206(42.1)$ & $143(46.3)$ & $63(35.0)$ & 0.0148 \\
\hline $\mathrm{BMI}, \mathrm{kg} / \mathrm{m}^{2}$ & $26.2 \pm 4.9$ & $25.9 \pm 4.8$ & $26.7 \pm 5.0$ & 0.0740 \\
\hline \multicolumn{5}{|l|}{ Cardiac risk factors } \\
\hline Diabetes mellitus, n (\%) & $130(26.6)$ & $78(25.2)$ & $52(28.9)$ & 0.3787 \\
\hline Hypercholesterolaemia, n (\%) & $303(62.0)$ & $187(60.5)$ & $116(64.4)$ & 0.3884 \\
\hline Arterial Hypertension, n (\%) & $417(85.3)$ & $259(83.8)$ & $158(87.8)$ & 0.2334 \\
\hline \multicolumn{5}{|l|}{ Past medical history } \\
\hline Previous MI, n (\%) & $72(14.7)$ & $48(15.5)$ & $24(13.3)$ & 0.5077 \\
\hline Previous PCI, n (\%) & $119(24.3)$ & $82(26.5)$ & $37(20.6)$ & 0.1371 \\
\hline Previous CABG, n (\%) & $35(7.2)$ & $17(5.5)$ & $18(10.0)$ & 0.0627 \\
\hline Previous stroke or TIA, n (\%) & $33(7.0)$ & $14(4.8)$ & $19(10.8)$ & 0.0136 \\
\hline $\begin{array}{l}\text { Peripheral vascular disease, } \\
\text { n (\%) }\end{array}$ & $70(14.3)$ & $50(16.2)$ & $20(11.1)$ & 0.1226 \\
\hline $\begin{array}{l}\text { Chronic obstructive pulmonary } \\
\text { disease, n (\%) }\end{array}$ & $80(16.4)$ & $56(18.1)$ & $24(13.3)$ & 0.1673 \\
\hline \multicolumn{5}{|l|}{ Clinical features } \\
\hline hypertension, n (\%) & $417(85.3)$ & $259(83.8)$ & $158(87.8)$ & 0.2334 \\
\hline \multicolumn{5}{|l|}{ Renal failure } \\
\hline $\mathrm{n}(\%)$ & $337(69.1)$ & $218(70.8)$ & $119(66.1)$ & 0.2818 \\
\hline Coronary artery disease, n (\%) & $297(60.7)$ & $191(61.8)$ & $106(58.9)$ & 0.5232 \\
\hline Atrial fibrillation, n (\%) & $156(31.9)$ & $100(32.4)$ & $56(31.1)$ & 0.7746 \\
\hline $\begin{array}{l}\text { Prior permanent pacemaker, } \\
\mathrm{n}(\%)\end{array}$ & $45(9.2)$ & $34(11.0)$ & $11(6.1)$ & 0.0711 \\
\hline $\begin{array}{l}\text { Calcification score, mm3; } \\
\text { median (IQR) }\end{array}$ & $\begin{array}{c}259 \\
(129-466)\end{array}$ & $\begin{array}{c}290 \\
(125-484)\end{array}$ & $\begin{array}{c}246 \\
(134-400)\end{array}$ & 0.5139 \\
\hline \multicolumn{5}{|l|}{ Symptoms } \\
\hline NYHA Functional Class & & & & 0.5640 \\
\hline $\mathrm{I}, \mathrm{n}(\%)$ & $33(6.8)$ & $19(6.2)$ & $14(7.8)$ & \\
\hline II, n (\%) & $121(24.8)$ & $81(26.3)$ & $40(22.2)$ & \\
\hline III, n (\%) & $276(56.6)$ & $169(54.9)$ & $107(59.4)$ & \\
\hline IV, n (\%) & $58(11.9)$ & $39(12.7)$ & $19(10.6)$ & \\
\hline \multicolumn{5}{|l|}{ Risk assessment } \\
\hline Logistic EuroScore, $\%$ & $22.3 \pm 13.7$ & $23.5 \pm 14.8$ & $20.2 \pm 11.4$ & 0.0113 \\
\hline STS score, $\%$ & $6.8 \pm 4.4$ & $6.9 \pm 4.8$ & $6.6 \pm 3.5$ & 0.4705 \\
\hline \multicolumn{5}{|l|}{ Antithrombotic therapy at baseline } \\
\hline Aspirin, n (\%) & $295(60.6)$ & $188(61.2)$ & $107(59.4)$ & 0.6959 \\
\hline Clopidogrel, n (\%) & $96(19.7)$ & $59(19.2)$ & $37(20.6)$ & 0.7203 \\
\hline Oral anticoagulation, n (\%) & $129(26.5)$ & $78(25.4)$ & $51(28.3)$ & 0.4800 \\
\hline
\end{tabular}


449 Table 2: Echocardiographic imaging characteristics.

450 Pre- and post TAVI assessments via transthoracic echocardiography. Depicted are means \pm SD with

451 p-values from t-tests or counts (\%) with p-values from Fisher's tests (two categories) or chi-square

452 tests (more than two categories). LV = Left ventricle; TAVI= Transcatheter aortic valve implantation.

\begin{tabular}{|c|c|c|c|c|}
\hline & \multirow{2}{*}{$\frac{\text { Overall }}{\mathrm{N}=489}$} & \multirow{2}{*}{$\begin{array}{l}\text { Medtronic CoreValve } \\
\mathrm{N}=309\end{array}$} & \multirow{2}{*}{$\frac{\text { Edwards Sapien }}{\mathrm{N}=180}$} & \multirow[t]{2}{*}{ Pvalue } \\
\hline & & & & \\
\hline \multicolumn{5}{|l|}{ Pre-TAVI assessment } \\
\hline Aortic valve area, $\mathrm{cm}^{2}$ & $0.70 \pm 0.23$ & $0.69 \pm 0.23$ & $0.72 \pm 0.22$ & 0.0901 \\
\hline Indexed aortic valve area, $\mathrm{cm}^{2} / \mathrm{m}^{2}$ & $0.39 \pm 0.12$ & $0.38 \pm 0.13$ & $0.40 \pm 0.12$ & 0.1289 \\
\hline Mean gradient, $\mathrm{mm} \mathrm{Hg}$ & $43.70 \pm 17.60$ & $44.16 \pm 18.42$ & $42.99 \pm 16.28$ & 0.5155 \\
\hline Peak gradient, mm Hg & $71.39 \pm 26.98$ & $72.02 \pm 27.89$ & $70.26 \pm 25.35$ & 0.5958 \\
\hline \multicolumn{5}{|l|}{ Left ventricular assessment } \\
\hline LV ventricular mass index $\left(\mathrm{g} / \mathrm{m}^{2}\right)$ & $152.14 \pm 42.03$ & $153.50 \pm 47.00$ & $151.13 \pm 38.47$ & 0.8112 \\
\hline LV endd iastolic diameter (LVEDD, mm) & $48.19 \pm 9.46$ & $48.03 \pm 10.63$ & $48.33 \pm 8.44$ & 0.8829 \\
\hline LV endsystolic diameter (LVESD, mm) & $32.80 \pm 10.60$ & $33.42 \pm 11.65$ & $32.21 \pm 9.63$ & 0.6281 \\
\hline \multicolumn{5}{|l|}{ Right ventricular assessment } \\
\hline Decreased right ventricular function & $26(21.5)$ & $15(25.4)$ & $11(17.7)$ & 0.3038 \\
\hline Severe pulmonary hypertension & $42(28.2)$ & $28(29.5)$ & $14(25.9)$ & 0.6436 \\
\hline \multicolumn{5}{|l|}{ Post-TAVI assessment } \\
\hline \multicolumn{5}{|l|}{ Aortic stenosis severity } \\
\hline Aortic valve area, $\mathrm{cm}^{2}$ & $1.84 \pm 0.56$ & $1.81 \pm 0.49$ & $1.88 \pm 0.62$ & 0.2658 \\
\hline Indexed aortic valve area, $\mathrm{cm}^{2} / \mathrm{m}^{2}$ & $1.02 \pm 0.32$ & $1.01 \pm 0.29$ & $1.03 \pm 0.34$ & 0.5163 \\
\hline Mean gradient, $\mathrm{mm} \mathrm{Hg}$ & $9.43 \pm 4.70$ & $8.85 \pm 4.75$ & $10.25 \pm 4.52$ & 0.0033 \\
\hline Peak gradient, mm Hg & $17.18 \pm 9.18$ & $16.23 \pm 9.40$ & $18.59 \pm 8.69$ & 0.0277 \\
\hline \multicolumn{5}{|l|}{ Left ventricular assessment } \\
\hline LV ejection fraction, \% & $56.50 \pm 11.87$ & $55.55 \pm 12.43$ & $57.83 \pm 10.94$ & 0.0543 \\
\hline LV ventricular mass index $\left(\mathrm{g} / \mathrm{m}^{2}\right)$ & $153.55 \pm 87.81$ & $152.70 \pm 45.45$ & $154.43 \pm 116.71$ & 0.8926 \\
\hline LV endd iastolic diameter (LVEDD, mm) & $46.90 \pm 8.45$ & $47.20 \pm 9.10$ & $46.57 \pm 7.69$ & 0.6079 \\
\hline LV endsystolic diameter (LVESD, mm) & $30.82 \pm 9.10$ & $31.52 \pm 10.14$ & $29.95 \pm 7.58$ & 0.2675 \\
\hline \multicolumn{5}{|l|}{ Right ventricular assessment } \\
\hline
\end{tabular}


456 Figure 1: (A) Cumulative incidence including landmark analysis of all-cause mortality according to

457 transcatheter aortic valve type up to 5 years of follow-up. (B) Cumulative incidence including

458 landmark analysis of cardiac mortality according to transcatheter aortic valve type up to 5 years of

459 follow-up. (C) Cumulative incidence including landmark analysis of major stroke according to

460 transcatheter aortic valve type up to 5 years of follow-up.

461 Medtronic CoreValve (blue line), Edwards Sapien (red line). (For interpretation of the references to colour in this figure legend, the reader is referred to the web version of this article.)

(A)

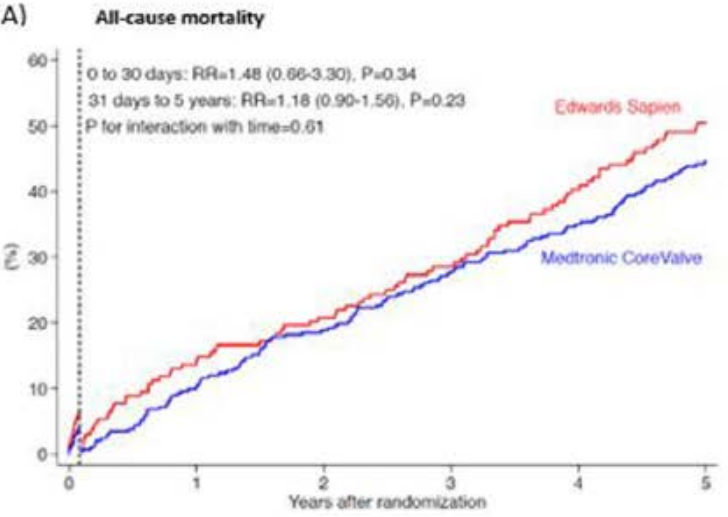
No at risk
Edwiuds Socien
Medtronic Conelavo

(C)

263

C) Major stroke

01030 days: RA-1.04 (037-289), P=0.94

31 days 105 yesr: RR=1.12 (0.36-3.41), P=0.85

8. P for interaction weth imo $=0.93$

¿

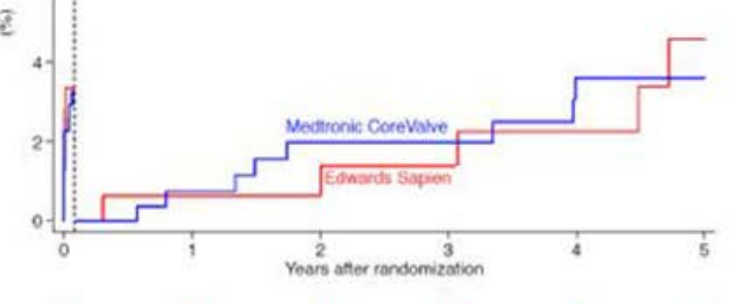


464 Figure 2: Cumulative incidence of structural valve deterioration up to 5 years of follow-up.

465 Medtronic CoreValve (blue line), Edwards Sapien (red line). (For interpretation of the references to 466 colour in this figure legend, the reader is referred to the web version of this article.)

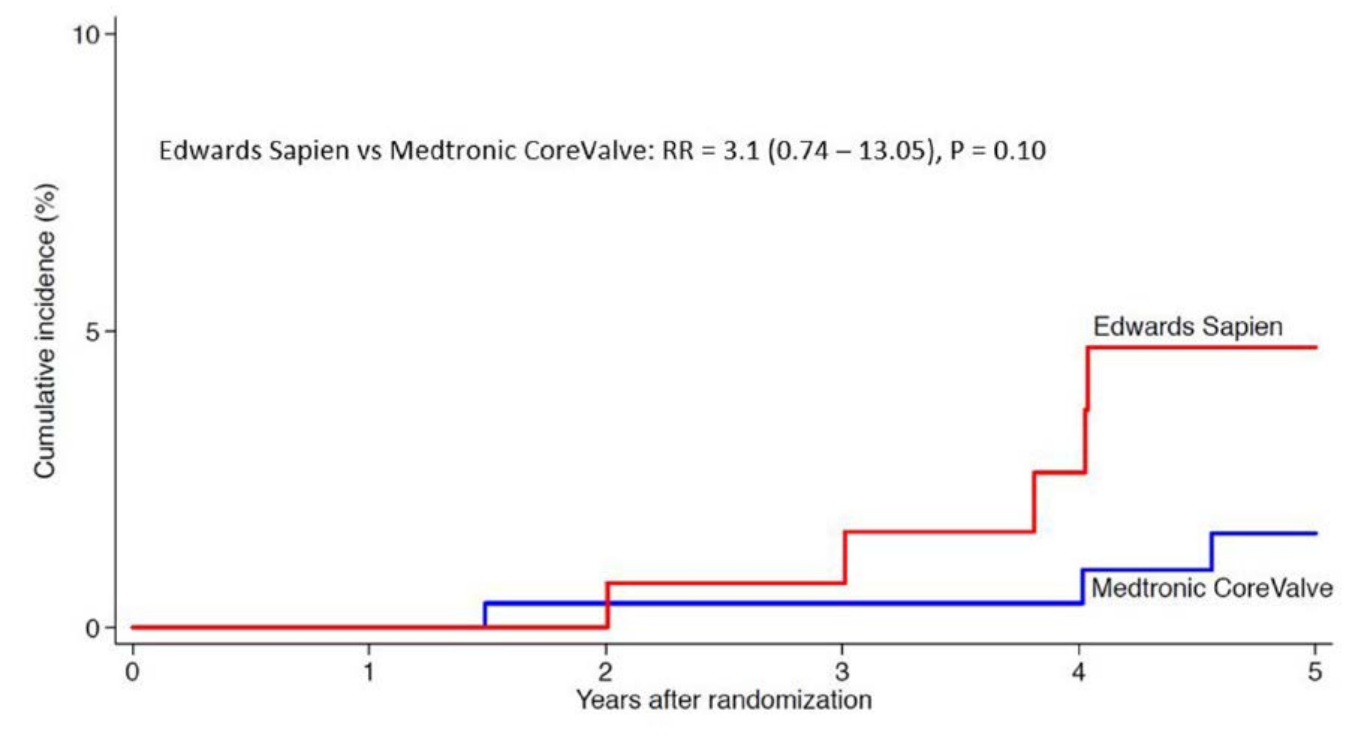

